\title{
Generalized Two-Dimensional Model Seismology with Application to Anisotropic Earth Models ${ }^{1}$
}

\author{
M. Nafi Toksöz and Don L. Anderson \\ Seismological Laboratory \\ California Institute of Technology, Pasadena
}

\begin{abstract}
The theory of two-dimensional seismic modeling is generalized to include the effect of anisotropy. The elastic coefficient matrix for a plate with orthorhombic symmetry is derived and is used to convert three-dimensional anisotropic problems into corresponding two-dimensional model problems. This is equivalent to replacing directional body velocities by the directional plate velocities. In addition to the application to seismic modeling, this can be considered a contribution to the basic theory of long waves in anisotropic plates. As such it has application to such problems as long waves in floating ice sheets. A model consisting of an anisotropic layer over an anisotropic half-space is constructed using a formica laver and a grooved aluminum plate. It is shown that rolled metal sheets can be made appreciably anisotropic by machining grooves in the surface. The experimental Rayleigh wave phase velocities are compared with the theoretical dispersion curves computed using isotropic and anisotropic theories. Two-layer circular models of the earth, one with an isotropic and the other with an anisotropic upper mantle, are fabricated, and a comparative study of body and surface waves is made. It is found that the relative effect of anisotropy is greater on surface waves than on body waves.
\end{abstract}

\section{INTRODUCTION}

Two-dimensional seismic models have played an important role in the study of elastic wave propagation in the past decade, and their use is now a basic seismological tool. Oliver et al. [1954] demonstrated by the use of Love's concept of 'generalized plane stress' that the plate equations of motion were exactly equivalent to the three-dimensional equations when the body velocities were replaced by the corresponding plate velocities. They also showed that the period equation for edge waves in a two-dimensional half-space was the same as the equation for classical Rayleigh waves, with plate velocities replacing body velocities. This equivalence holds for more complicated geometries, and many three-dimensional problems involving body and surface waves in isotropic mediums have been modeled exactly in two dimensions. Healy and Press [1960] extended the theory to composite plates and described fabrication of layered models. This technique makes it possible to model any velocity and density structure in two dimensions, provided the practical difficulties are eliminated.

\footnotetext{
${ }^{1}$ Contribution 1112, Division of Geological Sciences, California Institute of Technology, Pasadena.
}

Recent developments concerming the effects of anisotropy in wave propagation make it desirable to generalize further the two-dimensional theory to layered mediums with direetional velocities. In the first half of this paper such a generalized theory is developed. In the second half, the fabrication of anisotropic models and the results of experiments on layered, plane, and circular anisotropic models are described.

\section{MODEL THEORY}

The technique used in developing the original two-dimensional model theory was to utilize the stress components determined by Love from his concept of 'generalized plane stress.' These stresses were used in deriving the displacement equations of motion and the boundary conditions pertinent to wave propagation in a plate. Standard techniques were then applied in order to determine wave equations or perod equations, which turned out to be the same as the classical infinite body equations with plate velocities replacing the body velocities. In this paper we derive the isotropic model theory by an alternative approach and then generalize the theory to mediums with directional properties. The method is to develop a correspondence between the stress-strain matrix for a plate and 
for an infinite body. In addition to the seismic model applications, this theory has relevance to the study of long waves in such naturally occurring plates as floating ice sheets.

Let us consider elastic waves of the form

$$
\begin{array}{r}
(u, w)=\left[U_{i}(z), W_{i}(z)\right] e^{i(\omega t-k x)} \\
v=0, \frac{\partial(u, w)}{\partial y}=0
\end{array}
$$

traveling in the $X$ direction with particle motion in the $X Z$ plane.

The stress equations of motion are

$$
\begin{aligned}
& \partial p_{x x} / \partial x+\partial p_{x z} / \partial z=\rho \ddot{u} \\
& \partial p_{z x} / \partial x+\partial p_{z z} / \partial z=\rho \ddot{w}
\end{aligned}
$$

where the $p_{i j}$ are the stresses and the dots represent time derivatives. The boundary conditions are continuity of displacements and stresses throughout the medium except at free surfaces where the stresses vanish. The stresses are determined from the generalized Hook's law

$$
p_{i j}=C_{i j k l} e_{k l}
$$

where the $e_{k l}$ are elastic strains and the $C_{i j k l}$ are the elastic coefficients.

Thus far the discussion has been quite general, and it applies to isotropic, anisotropic, three-dimensional, and two-dimensional (plate) problems. Each of these problems must satisfy the same stress equations of motion and the same continuity conditions. Specialization occurs only when the form of the $C_{i j k l}$ in (3) has been assigned. This determines the form of the displacement equations of motion and the boundary conditions.

Indicial notation, i.e. $C_{i j k l} \rightarrow C_{a b}$, can be used to write the stress-strain relations in an infinite isotropic solid:

$$
\left[\begin{array}{l}
p_{x x} \\
p_{y y} \\
p_{z z} \\
p_{y z} \\
p_{x z} \\
p_{x y}
\end{array}\right]=\left[\begin{array}{cccccc}
C_{11} & C_{12} & C_{12} & 0 & 0 & 0 \\
C_{12} & C_{11} & C_{12} & 0 & 0 & 0 \\
C_{12} & C_{12} & C_{11} & 0 & 0 & 0 \\
0 & 0 & 0 & C_{44} & 0 & 0 \\
0 & 0 & 0 & 0 & C_{44} & 0 \\
0 & 0 & 0 & 0 & 0 & C_{44}
\end{array}\right]\left[\begin{array}{c}
e_{x x} \\
e_{y y} \\
e_{z z} \\
e_{y z} \\
e_{x z} \\
e_{x y}
\end{array}\right]
$$

where

$C_{44}=\left(C_{11}-C_{12}\right) / 2 ; C_{11}=\lambda+2 \mu ; C_{12}=\lambda$.

Now let us consider a free plate occupying the $X Z$ plane. The thin plate condition, $p_{y y}=$ 0 , substituted in (4), yields

$$
e_{u y}=-\frac{C_{12}}{C_{11}}\left[e_{x x}+e_{z z}\right]
$$

Equation 4 can now be written

$$
\left[\begin{array}{l}
p_{x x} \\
p_{z z} \\
p_{y z} \\
p_{x z} \\
p_{x y}
\end{array}\right]=\left[\begin{array}{ccccc}
C_{11}^{\prime} & C_{12}^{\prime} & 0 & 0 & 0 \\
C_{12}^{\prime} & C_{11}^{\prime} & 0 & 0 & 0 \\
0 & 0 & C_{44}^{\prime} & 0 & 0 \\
0 & 0 & 0 & C_{44} & 0 \\
0 & 0 & 0 & 0 & C_{44}
\end{array}\right]\left[\begin{array}{c}
e_{x x} \\
e_{z z} \\
e_{y z} \\
e_{x z} \\
e_{x y}
\end{array}\right]
$$

where

$$
C_{11}^{\prime}=C_{11}-C_{12}{ }^{2} / C_{11}
$$

and

$$
C_{12}^{\prime}=C_{12}-C_{12}{ }^{2} / C_{11}
$$

Equation 6 is the stress-strain matrix for a plate. It can be used in place of (4) to transform any three-dimensional isotropic problem to the equivalent two-dimensional model problem. This substitution simply replaces primed (plate) elastic coefficients with the unprimed (three-dimensional) elastic constants. In terms of the more familiar Lamé constants and elastic velocities,

$$
\begin{gathered}
\lambda^{\prime}=2 \mu \lambda /(\lambda+2 \mu) \\
\mu^{\prime}=\mu \\
\alpha^{\prime}=\sqrt{\frac{\lambda^{\prime}+2 \mu^{\prime}}{\rho}}=\sqrt{\frac{4 \mu(\lambda+\mu)}{\rho(\lambda+2 \mu)}} \\
\beta^{\prime}=\sqrt{\frac{\mu^{\prime}}{\rho}}=\sqrt{\frac{\mu}{\rho}}=\beta
\end{gathered}
$$

Note that $\mu$ and $\beta$ are unchanged by the transformation. The $\alpha^{\prime}$ is the classical plate velocity.

Let us now consider a solid with three mutually perpendicular axes of elastic symmetry. The elastic coefficient matrix becomes 


$$
C_{a h}=\left[\begin{array}{cccccc}
C_{11} & C_{12} & C_{13} & 0 & 0 & 0 \\
C_{12} & C_{22} & C_{23} & 0 & 0 & 0 \\
C_{13} & C_{23} & C_{33} & 0 & 0 & 0 \\
0 & 0 & 0 & C_{44} & 0 & 0 \\
0 & 0 & 0 & 0 & C_{55} & 0 \\
0 & 0 & 0 & 0 & 0 & C_{60}
\end{array}\right]
$$

For a plane wave traveling in the $X Z$ plane in a direction specified by direction cosines $l$ and $n$, the displacements are

$$
(u, w)=(U, W) e^{i \omega t} e^{-k(l x+n z)}
$$

Substitution into the equations of motion yields the velocity equation

$$
\begin{gathered}
{\left[\left(l^{2} C_{11}+n^{2} C_{55}-\rho c^{2}\right)\left(l^{2} C_{55}+n^{2} C_{33}-\rho c^{2}\right)\right.} \\
\left.-n l\left(C_{13}+C_{55}\right)\right]=0
\end{gathered}
$$

from which the velocity in any direction can be determined.

In the $X$ direction, $l=1, n=0$, and we obtain the two velocities

$$
\begin{aligned}
& \rho \alpha_{1}{ }^{2}=C_{11} \\
& \rho{\beta_{1}}^{2}=C_{55}
\end{aligned}
$$

In the $Z$ direction, $l=0, n=1$, and

$$
\begin{aligned}
& \rho \alpha_{2}{ }^{2}=C_{33} \\
& \rho \beta_{2}{ }^{2}=C_{55}
\end{aligned}
$$

In these two directions the roots of the velocity equation represent purely longitudinal and purely transverse motion. This is not, in general, true for other directions.

To describe motion completely in the $X Z$ plane, the constant $C_{13}$ is required. It can be recovered from a measurement in any direction other than $X$ or $Z$ using equation 11 .

Let us now consider a plate in the $X Z$ plane. The following analysis, with the appropriate change of constants, holds equally well for a plate in the $X Y$ or the $Y Z$ plane. A rolled or extruded plate, a fibrous sheet, or a prestressed floating ice sheet would be expected to display this kind of anisotropy.

Again, the condition of plane stress is $p_{y y}=$ 0 , yielding

$$
e_{y y}=-\frac{C_{12} e_{x x}+C_{23} e_{z z}}{C_{22}}
$$

This substitution in (9) gives the plate matrix

$$
C_{a b}^{\prime}=\left[\begin{array}{ccccc}
C_{11}^{\prime} & C_{13}^{\prime} & 0 & 0 & 0 \\
C_{13}^{\prime} & C_{33}^{\prime} & 0 & 0 & 0 \\
0 & 0 & C_{44} & 0 & 0 \\
0 & 0 & 0 & C_{55} & 0 \\
0 & 0 & 0 & 0 & C_{66}
\end{array}\right]
$$

where

$$
\begin{gathered}
C_{11}{ }^{\prime}=C_{11}-C_{12}{ }^{2} / C_{22} \quad C_{33}{ }^{\prime}=C_{33}-C_{23}{ }^{2} / C_{22}{ }^{2} \\
C_{13}{ }^{\prime}=C_{13}-C_{23} C_{12} / C_{22}
\end{gathered}
$$

The elastic constants pertinent to the problem of body and Rayleigh-type surface wave propagation in the $X Z$ plane are $C_{11}, C_{13}, C_{39}$, and $C_{55}$ and, for plates, their primed equivalents. Four elastic constants are sufficient, also, for describing this type of propagation along any other axial direction. The problem of elastic wave propagation along any axial direction in an orthorhombic medium can therefore be reduced to the corresponding problem for a hexagonal, or transversely isotropic, medium. The solutions given by Anderson [1961] are therefore pertinent to the present problem when $C_{44}$ is replaced by $C_{55}$. The problem of body and surface waves in layered transversely isotropic medium can be modeled in two dimensions by the substitutions of equation 14. Note that the directional plate velocities are

$$
\begin{array}{ll}
\alpha_{1}^{\prime}=\sqrt{C_{11}^{\prime} / \rho} & \alpha_{2}^{\prime}=\sqrt{C_{33}^{\prime} / \rho} \\
\beta_{1}^{\prime}=\beta_{1} & \beta_{2}{ }^{\prime}=\beta_{2}
\end{array}
$$

$$
\left.\left(\begin{array}{l}
\alpha_{3}{ }^{\prime} \\
\beta_{3}{ }^{\prime}
\end{array}\right)=\left[\frac{\frac{\left(C_{11^{\prime}}+C_{33^{\prime}}+2 C_{55}\right)}{2} \pm\left[\left(\frac{C_{11}{ }^{\prime}-C_{33}{ }^{\prime}}{2}\right)^{2}+\left(C_{13}^{\prime}+C_{55}\right)^{2}\right.}{2 \rho}\right]^{1 / 2}\right]^{1 / 2}
$$


where $\alpha_{s}^{\prime}$ is the faster plate wave traveling at $45^{\circ}$ to the $X$ and $Z$ axes and $\beta_{3}^{\prime}$ is the slower. Neither of these waves is purely shear or purely compressional but represent coupled motion.

\section{Experimint}

Two diferent two-dimensional nodels were used in studying the effects of anisotropy on elastic wave propagation. Naturally anisotropic materials and materials made anisotropic by artificial processes were used. Most plastics and nonmetals suitable for two-dimensional modeling have drectional elastic properties, and in many cases this anisotropy exceds 10 per cent. However, the densities and acoustic velocities of these materials fall in a relatively narrow region, making it impractical to use more than one such plastic in fabricating a realistic two-dimensional layered model.

Metal sheets commonly used in seismio models are less than 1 or 2 per cent anisotropic. We measured velocities in several cold-rolled aluminum and steel sheets and were unable to find one sufficiently anisotropic. By grooving the sheet, however, we were able to introduce an appreciable directionality for the elastic parameters.

In a grooved plate where grooves and ridges form a periodic structure, the velocities in different directions for long wavelengths can be computed by physical reasoning. The solution of the appropriate boundary value problem for this case would be very difficult, if possible at all.

Let us consider a grooved plate as illustrated in Figure 1. In computing the elastic parameters of this plate we need to make the following assumptions: 1. The thickness of the plate, $t$, is much smaller than the shortest wavelengths encountered in the experiment. In other words, the plate is an ideal one, the plate velocities are independent of the thickness, and the stress fields are uniform. 2. The width of the grooves is less than the thickness.

The velocity of longitudinal waves in the direction parallel to the grooves, $\alpha_{1}$, is the same as that of the ungrooved plate becanse of the thin plate approximation. In the direction transverse to the grooves a new plate velocity arises because of the increase in compressional stress as the plane wave propagates from the ridge into the grooved section. One can compute an effective Young's modulus $\left(E_{z}\right)_{\text {ex }}$ for the combina-

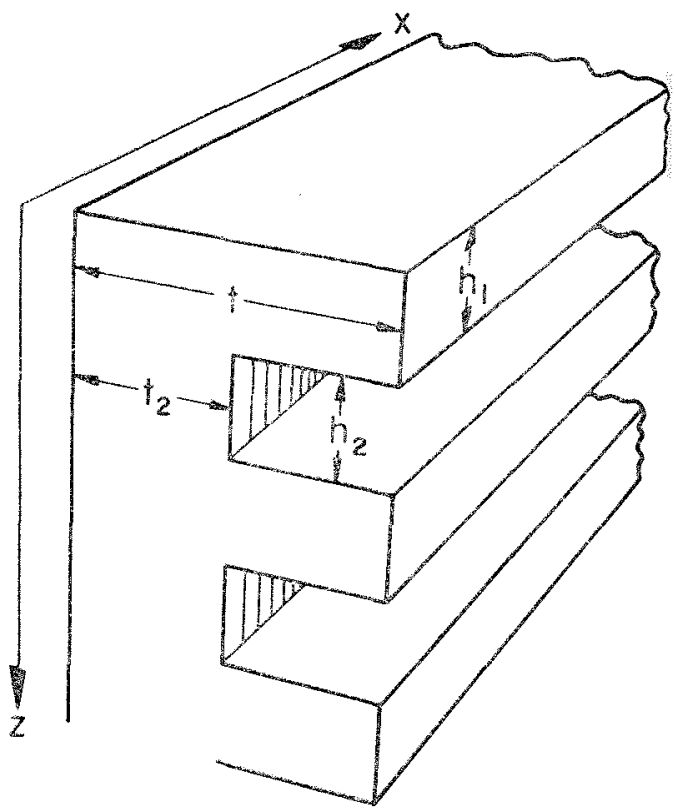

Fig. 1. Geometry of grooved plate.

tion of one groove and a ridge in a plate of: thickness $t$ by taking the ratio of stress over total strain:

$$
\left(H_{z}\right)_{\mathrm{or}}=\frac{\sigma_{0}\left(h_{1}+h_{2}\right)}{e_{0} h_{1}+e_{2} h_{2}}
$$

where $\sigma_{0}$ and $e_{0}$ are stress and strain, respectively, in the ungrooved section. Also $h_{1}$ and $h_{2}$ are the respective depths and $t$ and $t_{2}$ are the respective thicknesses, as shown in Figure 1 . Substituting $e_{2}=\left(t / t_{2}\right) e_{0}$ into (16) we obtain

$\left(E_{2}\right)_{\mathrm{elf}}=\frac{\sigma_{0}}{e_{0}} \frac{h t_{2}}{h_{1} t_{2}+h_{2} t}=E_{0} \frac{h t_{2}}{h_{1} t_{2}+h_{2} t}$

where $E_{0}=$ Young's modulus for the ungrooved plate and $h=h_{1}+h_{3}$.

The same argument also holds for the rigidity. Because of the transverse nature of the motion in the shear waves, the effective rigidities in both $X$ and $Z$ directions are given by expressions equivalent to (17). When the groove width is equal to the ridge width and when the groove depth is half the plate thickness, $h_{1}=h_{: 2}=h / 2$ and $t_{\mathrm{s}}=t / 2$, then,

$$
\left(E_{z}\right)_{\text {eff }}=\frac{2}{3} E_{0} \quad\left(\mu_{x}\right)_{\text {eff }}=\left(\mu_{z}\right)_{\text {eff }}=\frac{2}{3} \mu_{0}
$$

The $P$ and $S$ velocities are 
$\alpha_{2}=\sqrt{\frac{2}{3}} \alpha_{0} \quad \beta_{1}=\beta_{2}=\sqrt{\frac{2}{3}} \beta_{0}$

From the velocities along and perpendicular to the grooves three of the four moduli necessary to compute velocities in any direction can be obtained. The fourth modulus requires the velocity along a direction other than $X$ and $Z$ directions.

Anisotropic layer over anisotropic half-space. A formica layer and a grooved aluminum plate were joined to make this model. The aluminum plate is $1 / 8$ inch thick, and the grooves are $1 / 16$ inch deep and $1 / 16$ inch wide. Compressional and shear velocities in radial directions between $\theta=0^{\circ}$ ( $X$ direction) and $\theta=90^{\circ}$ ( $Z$ direction) are at $5^{\circ}$ and $10^{\circ}$ intervals. The measured velocities and the theoretical ones computed using equation 18 are listed in Table 1 . The agreement is good, considering the approximations and assumptions. A polar graph of the measured compressional and shear velocities is shown in Figure 2. These graphs show maximums at $\Theta=0^{\circ}$ for the compressional velocities and at $\theta=45^{\circ}$ for the shear velocities.

Volocities of both body and the Rayleigh waves were measured along $\theta=0^{\circ}$ and $\theta=$ $90^{\circ}$. The results, along with the theoretical valnes computed from the anisotropic theory [Andorson, 1961] using the measured body wave velocities, are given in Table 1 . The agreement is not perfect, and this is mainly due to errors that are introduced in computing effective elastic parameters from observed velocities which are not very accurate.

A sheet of formica $1 / 16$ inch thick was used
TABLIE 1. Measured and Computed Directional Velocities

\begin{tabular}{|c|c|c|c|}
\hline \multirow{2}{*}{ Wave } & \multirow{2}{*}{$\begin{array}{l}\text { Ungrooved } \\
1 / 8 \text {-Inch } \\
\text { Aluminum } \\
\text { Plate } \\
\text { Measured, } \\
\text { in./ } / \text { sec }\end{array}$} & \multicolumn{2}{|c|}{$\begin{array}{l}\text { Groved } \\
\text { Aluminum } \\
\text { Plate }\end{array}$} \\
\hline & & $\begin{array}{l}\text { Measured, } \\
\text { in. } / \mu \text { sec }\end{array}$ & $\begin{array}{l}\text { Computed, } \\
\text { in. } / \mu \mathrm{sec}\end{array}$ \\
\hline$\alpha_{1}\left(0^{\circ}\right)$ & 0.212 & 0.208 & 0.212 \\
\hline$\alpha_{2}\left(90^{\circ}\right)$ & 0.212 & 0.173 & 0.173 \\
\hline$\beta_{1}\left(0^{\circ}\right)$ & 0.121 & 0.106 & 0.059 \\
\hline$\beta_{2}\left(90^{\circ}\right)$ & 0.121 & 0.106 & 0.099 \\
\hline$C_{R}\left(0^{\circ}\right)$ & 0.099 & 0.101 & 0.099 \\
\hline$C_{R 2}\left(30^{\circ}\right)$ & 0.099 & 0.100 & 0.098 \\
\hline
\end{tabular}

for the layer portion of the model, and it was joined to grooved aluminum by an epoxy resin as shown in Figure 3. Before the construction was started, however, the velocities were measured along various polar angles. The polar diagram of $P$ and $S$ velocities is shown in Figure 2 , and the critical values are given in the table of Figure 3. The anisotropy of formica is due to its fiber structure. Unfortunately, this structure is neither completely uniform nor orthogonal, and this is reflected in measured values of polar velocity distribution.

Two different models having an anisotropic layer over an anisotropic half-space were constructed in order to measure the phase velocity of Rayleigh waves. In the first model the thickness of the formica layer was 1 inch. To obtain more data at the lower-frequency end of the

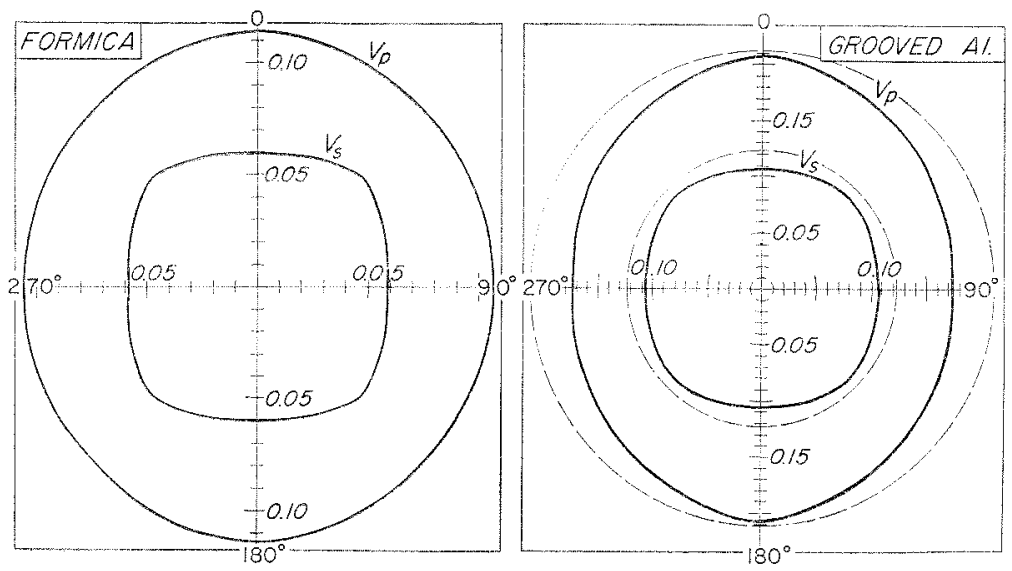

Fig. 2. Polar velocity diagrams for measured $P$ and $S$ velocities in anisotropic formica and grooved aluminum plate. The dashed circles refer to ungrooved isotropic aluminum. The $X^{\prime}$ axis is along $0^{\circ}$ and the $Z$ axis is along $90^{\circ}$. 


\begin{tabular}{|c|c|c|c|c|c|}
\hline & & $\begin{array}{c}a_{\mathrm{i}} \\
\text { NN } / \mu \text { SEC }\end{array}$ & $\begin{array}{c}\beta_{\mathrm{i}} \\
\mathrm{N} . / \mu \mathrm{SEC}\end{array}$ & $\begin{array}{c}\mathrm{C}_{\mathrm{R}} \\
\text { IN. } / \mu \mathrm{SEC}\end{array}$ & $\begin{array}{c}P_{i} \\
\text { GM.CM }{ }^{3}\end{array}$ \\
\hline \multirow{3}{*}{ FORMICA } & $0^{\circ}$ & 0.113 & 0.060 & 0.056 & \multirow{3}{*}{1.36} \\
\hline & $45^{\circ}$ & 0.108 & 0.068 & - & \\
\hline & $90^{\circ}$ & 0.106 & 0.060 & 0.056 & \\
\hline \multirow{3}{*}{$\begin{array}{l}\text { GROOVED } \\
\text { ALUMINUM }\end{array}$} & $0^{\circ}$ & 0.208 & 0.106 & 0.101 & \multirow{3}{*}{2.70} \\
\hline & $45^{\circ}$ & 0.188 & 0.113 & - & \\
\hline & $90^{\circ}$ & 0.173 & 0.106 & 0.100 & \\
\hline
\end{tabular}

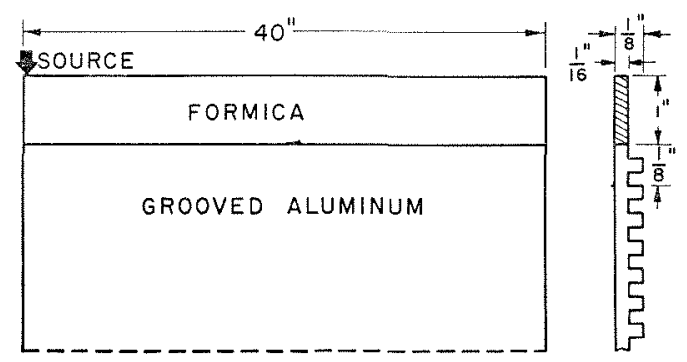

Fig. 3. Model having anisotropic formica layer over anisotropic grooved aluminum half-space. The table gives the measured elastic parameters in the layer and the half-space.

dispersion curve, another model was constructed, with the thickness of the formica layer reduced to $1 / 2$ inch. There were some difficulties in obtaining an uncontaminated dispersed Rayleigh wave train at longer periods, because of the extensive interference of $P L$ waves. The $P L$ waves are mainly due to the large reflection coefficient at the formica-aluminum interface. Figure $4(a)$ is a typical seismogram filtered by a low-pass filter, and it shows the fundamental nondegenerate $P L$ mode and the Rayleigh mode. [Phinney, 1961; Gilbert and Laster, 1962]. Separating the low-frequency Rayleigh wave and minimizing the interference of $P L$ waves require very careful choice of the velocity and frequency windows. Part $(b)$ of the figure shows a highpass-filtered record in which the $P_{n}$ and first higher mode of $P L, S_{n}$, and Rayleigh wave train are outstanding. In this case the Rayleigh train is well isolated from $P L$ interference.

The phase velocities were determined both from time domain records by matching troughs and peaks on narrow-band-filtered traces and from Fourier phase spectrums of the Rayleigh pulses, using the formula [Ben-Menahem and Toksöz, 1961]

$$
C(T)=\frac{\Delta x}{\Delta t+T(\Delta \varphi+n)}
$$

where $C^{\prime}$ is the phase velocity, $\triangle x$ is the distance between two stations, $\Delta t$ is the time difference between the beginning of windows, $T$ is the period, $\Delta \varphi$ is the phase difference, and $n$ is an integer. The measured phase velocities are shown in Figure 5.

Theoretical phase-velocity curves for this particular model were computed from both isotropic and anisotropic theory. For isotropic computations several velocity combinations (velocities along $\theta=0^{\circ}, \theta=45^{\circ}$, average, and maximum velocities) were used, since there is not a unique set. All of these theoretical curves failed to fit the experimental data. Two of these, one using maximum velocities $\left(\alpha_{0}, \beta_{45}\right)$ and the other using the velocities along $\theta=0^{\circ}$, are shown in Figure 5. The theoretical curve computed from anisotropic theory [Harkrider and Anderson, 1962] is shown as a dashed curve in the same figure. The agreement between the theoretical and the experimental is very good, especially at longer periods. The discrepancy at shorter periods is mainly due to the nonorthogonal symmetry of the anisotropic formica layer.

Circular layered earth models with isotropic and anisotropic upper mantle. The possible presence of an anisotropic zone in the upper part of the earth's mantle has great significance in the interpretation of seismological data. To make a comparative study, two identical earth models, one with an isotropic and the other with an anisotropic upper mantle, were constructed. The isotropic model is identical with that described by Healy and Press [1960]. It consists of a circular, aluminum disk 0.020 inch thick, with a radius of 12 inches. The outer first inch of the plate is milled, filled with plastic, and recontoured as shown in Figure 6. This gives two layers whose elastic parameter (compressional and shear velocities, density) ratios approximate those of the earth with a two-layered crust and a mantle. In scaling, the thicknesses of layers are exaggerated in comparison with the radius of the model to make it possible to take measurements within a convenient frequency band and yet keep the model size reasonable.

Both $P$ and $S$ wave travel times and Rayleigh wave phase velocities were measured on this model. After completion of measurements, the 


\section{$P_{1} P_{L 21} R$}

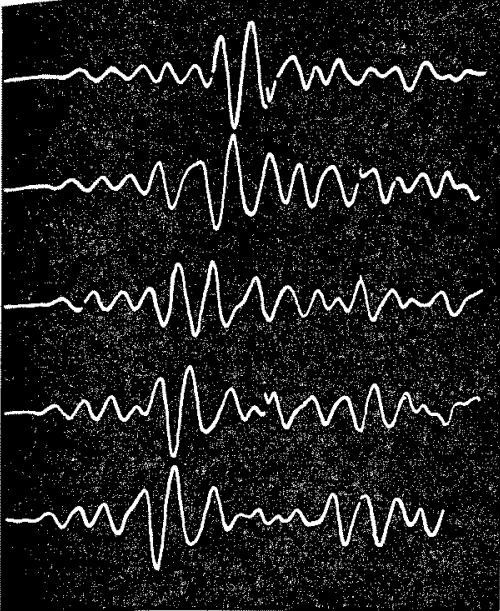

FILTER: $2^{\mathrm{K}}-20^{\mathrm{K}}$ (a)

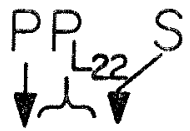

$R$

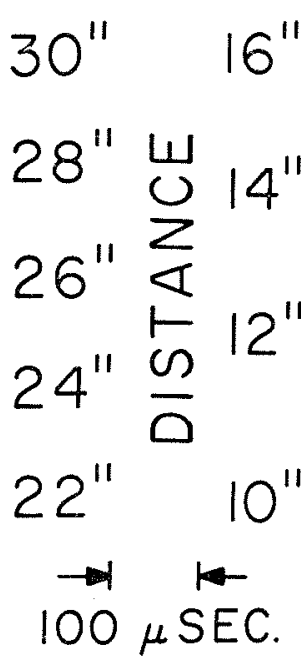

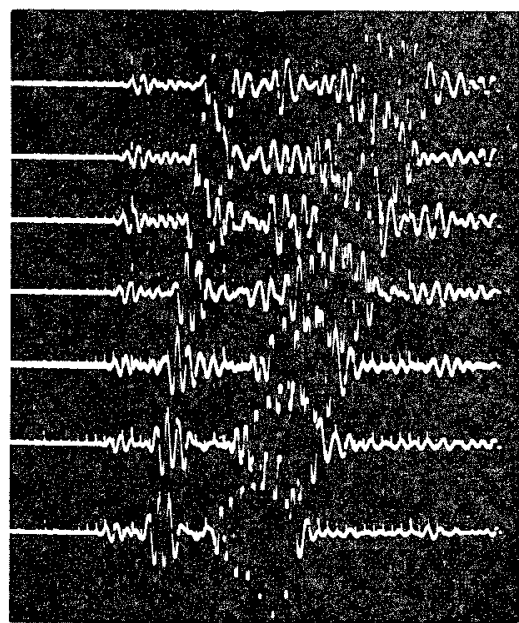

FILTER:100 $-200^{K}$ (b)

Fig. 4. Sample seismograms from anisotropic layer over anisotropic half-space model. Distances from the source are given in inches. (a) Band-pass filter, 2 to $20 \mathrm{kc} / \mathrm{s}$. Nole the dispersed fundamental PL mode. (b) Band-pass filter, 100 to $200 \mathrm{kc} / \mathrm{s}$. Higher mode of $P L$ wave, along with shear and Rayleigh modes, are outstanding.

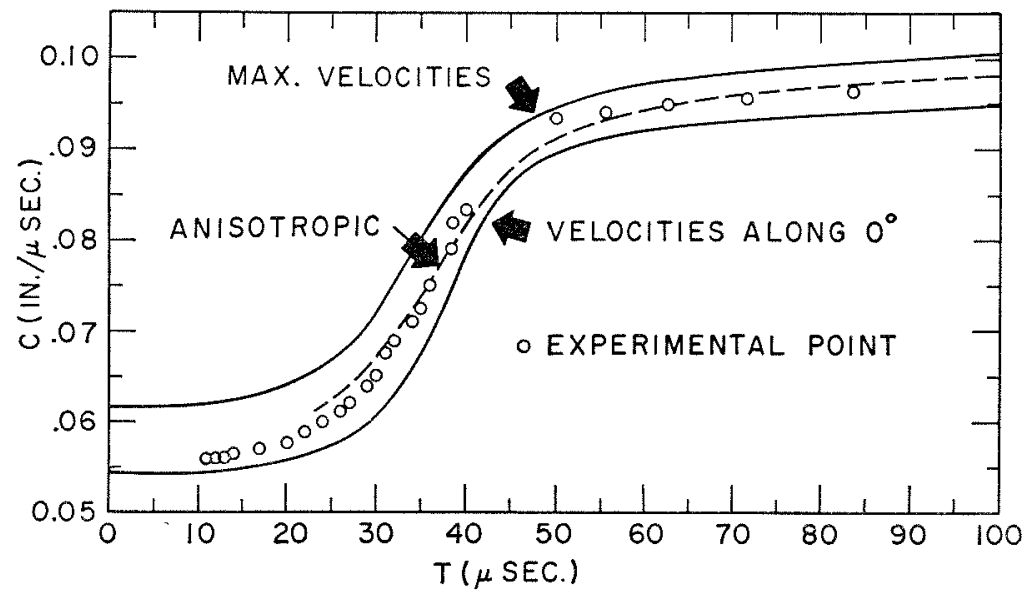

Fig. 5. Experimental and theoretical Rayleigh wave phase velocities for the anisotropic layer over anisotropic half-space. The solid curves are computed from isotropic theory using $P$ and $S$ velocities in the $X$ direction (refraction velocities) and the maximum velocities $\left[\alpha\left(0^{\circ}\right)\right.$ and $\left.\beta\left(45^{\circ}\right)\right]$. The dashed theoretical curve is computed from anisotropic theory, using the actual directional velocities. 

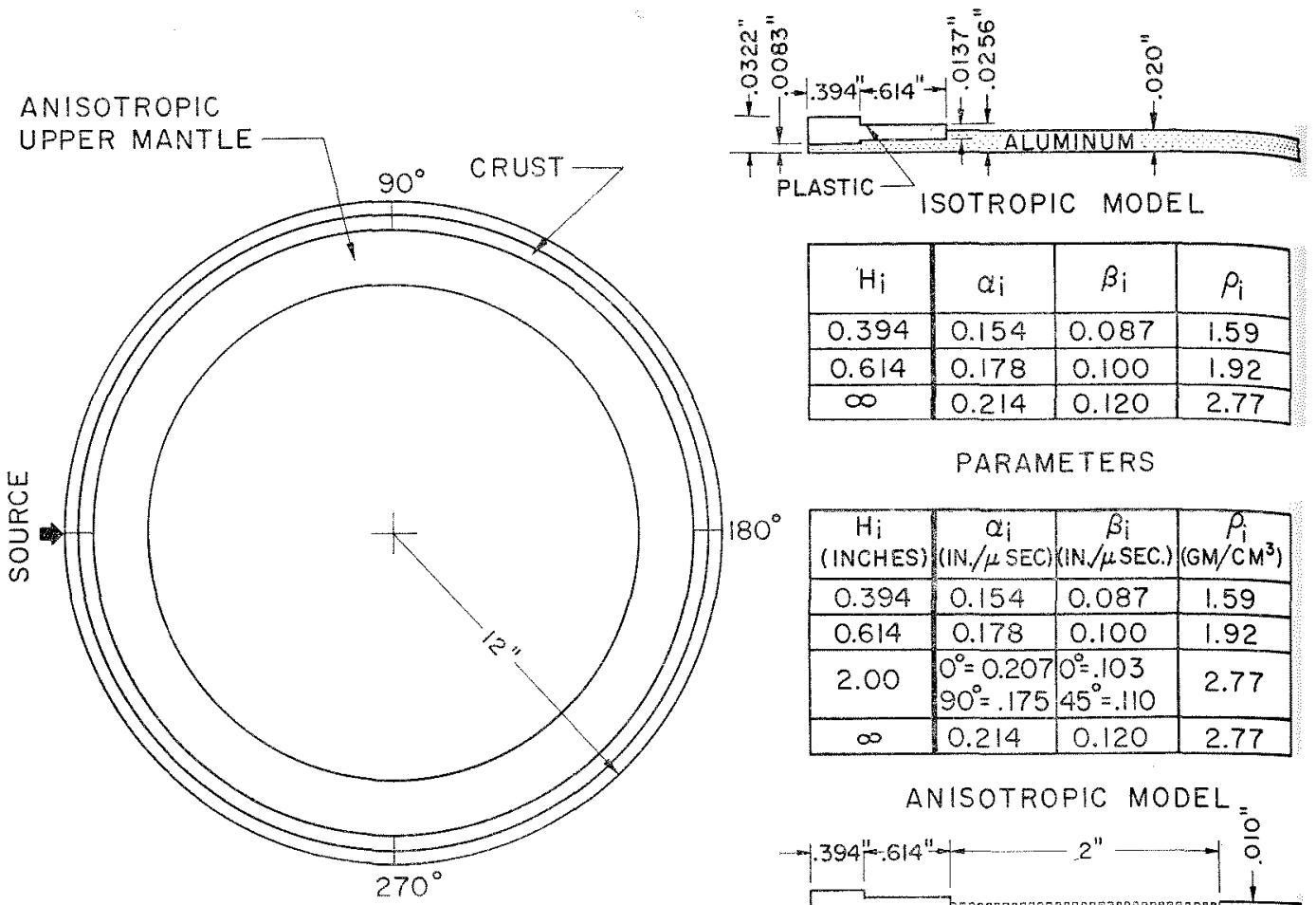

\begin{tabular}{|c|c|c|c|}
\hline$H_{i}$ & $\alpha_{i}$ & $\beta_{i}$ & $\rho_{i}$ \\
\hline 0.394 & 0.154 & 0.087 & 1.59 \\
\hline 0.614 & 0.178 & 0.100 & 1.92 \\
\hline$\infty$ & 0.214 & 0.120 & 2.77 \\
\hline
\end{tabular}

PARAMETERS

\begin{tabular}{|c|c|c|c|}
\hline $\begin{array}{c}\mathrm{Hi} \\
\text { (INCHES) }\end{array}$ & $\left(\begin{array}{c}\alpha i \\
(1 N . / \mu \mathrm{SEC})\end{array}\right.$ & $\begin{array}{c}\beta_{i} \\
(\mathbb{N} / \mu S E C .)\end{array}$ & $\begin{array}{c}\bar{P}_{i} \\
\left(G M / \mathrm{CM}^{3}\right)\end{array}$ \\
\hline 0.394 & 0.154 & 0.087 & 1.59 \\
\hline 0.614 & 0.178 & 0.100 & 1.92 \\
\hline 2.00 & $\begin{array}{l}0^{\circ}=0.207 \\
90^{\circ}=.175\end{array}$ & $\begin{array}{l}0^{\circ}=.103 \\
45^{\circ}=.110\end{array}$ & 2.77 \\
\hline$\infty$ & 0.214 & 0.120 & .77 \\
\hline
\end{tabular}

ANISOTROPIC MODEL.

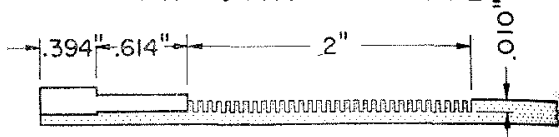

Fig. 6. Cireular layered earth models with isotropic and anisotropic upper mantle. The parameters of the isotropic and the anisotropic models are given in the tables.

outer 2 inches of the mantle portion of the model were grooved to a depth of 0.010 inch, with a spacing of 0.03125 inch. This gives a new model that is identical with the old one, except for the anisotropy introduced in the upper mantle by the grooving process. The parameters of both models are listed in the tables of Figure 6. The directional velocities of the anisotropic layer were computed using the measured velocity ratios of the grooved plane model described earlier in this paper, with the assumption that the same theory is cqually applicable here.

The same measurements were taken on the anisotropic model that were taken on the isotropic one. Figure 7 shows two records, one from each model, with identical filters and at identical distances. The $P, S$, and Rayleigh waves are indicated by arrows. Very little change in character can be noticed between two records for corresponding $P$ and $S$ waves; however, the variation is more outstanding for the Rayleigh waves.
Higure 8 shows the travel-time curves for both models. For $P$ waves the difference is barely visible at a distance of $90^{\circ}$ from the source. For $S$ waves this variation becomes observable at $45^{\circ}$. Also, the maximum variation in travel times between isotropic and anisotropic models takes place at different distances for $P$ and $S$ waves. This is also reasonable, since the directions of maximum velocities are different for the two waves. The over-all effect of anisotropy on travel-time curves would be to change the $(S-P)$ time in comparison with the isotropic model. This change, of course, is dependent on the nature of the anisotropy and on the distance from the source.

The effect of anisotropy on surface wave dispersion is illustrated in Figure 9, where experimental phase velocities are shown for both cases At periods shorter than $20 \mu \mathrm{sec}$, the two curves are identical, as they should be. These short wavelengths are controlled mostly by the crust which is the same in both models. As the periods 


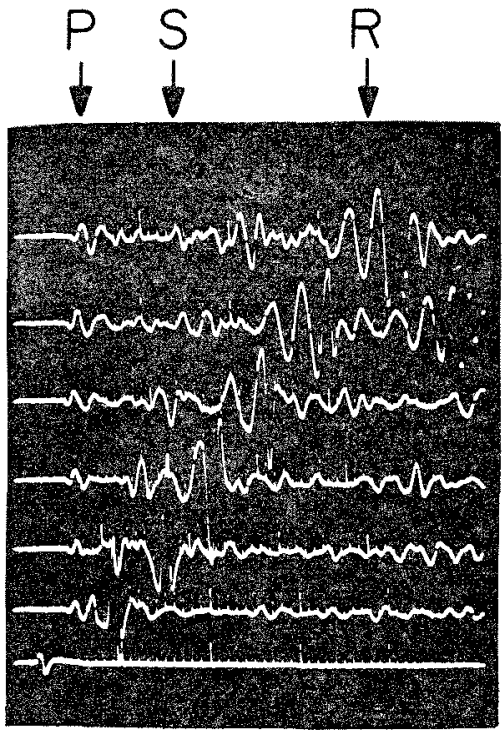

ISOTROPIC

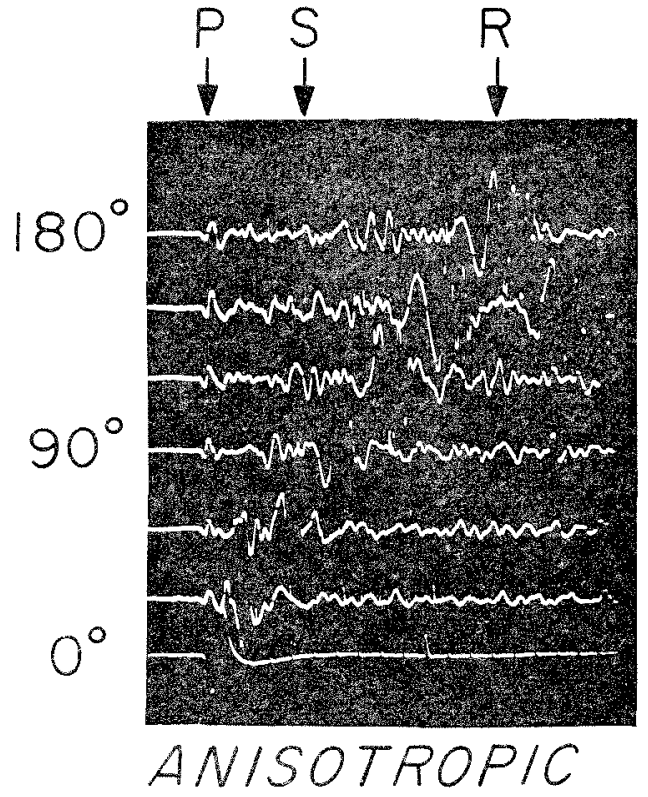

Fig. 7. Sample seismograms from the circular isotropic and anisotropic earth models. Distances from the source are given in degrees.

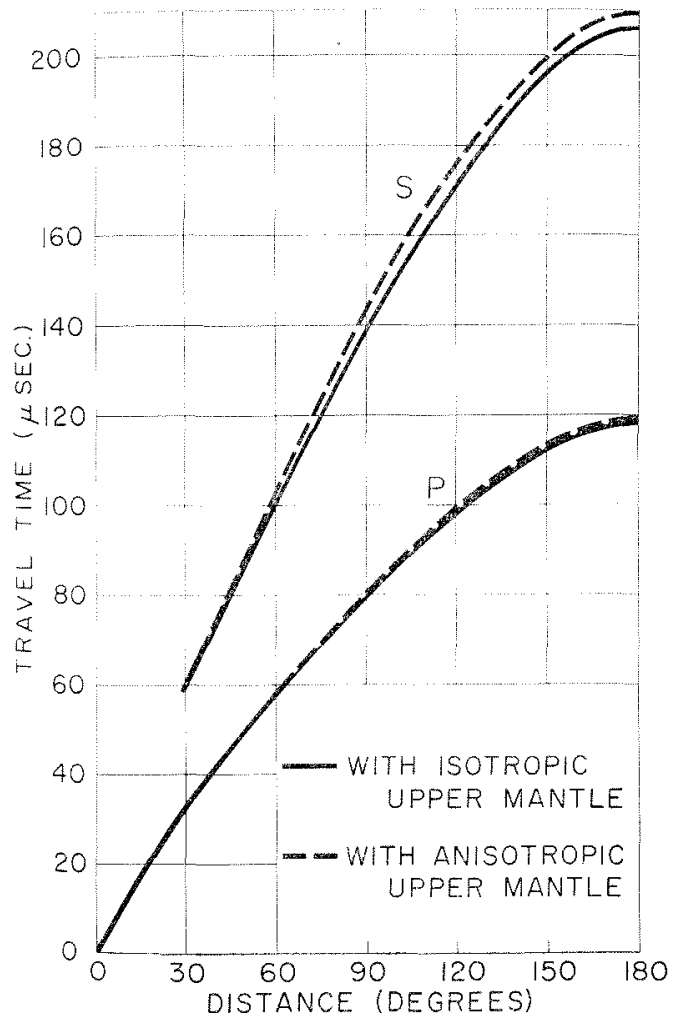

Fig. 8. $P$ and $S$ travel-time curves for the circulat earth models. increase, the waves are affected more and more by the mantle, and hence by the anisotropic zone. This gives rise to appreciable difference in phase velocities. No effort was made to fit theoretical curves to these data. Using the dispersion curve for a nonlayered cylindrical model [Ewing et $a l ., 1957, p .263]$ and correcting the curves by the same ratio works over a certain period range. This, however, is a rough approximation. At the present time, work is being done in our laboratory on curvature corrections that would be usable in all layered cases.

To get a qualitative estimate of the effect of

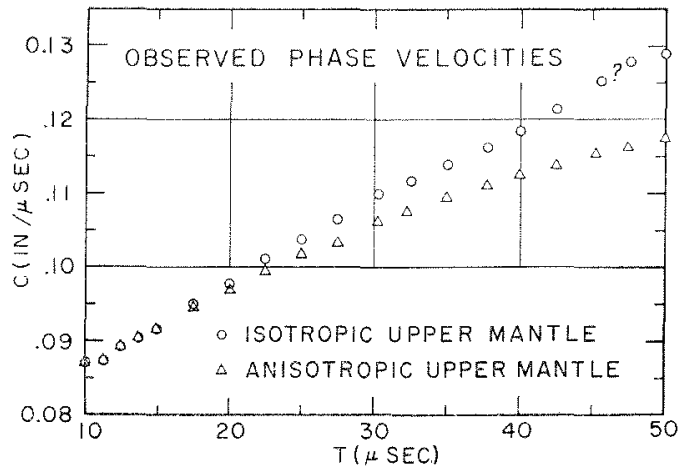

Fig. 9. Experimental Rayleigh wave dispersion cturves for the circular earth models 
anisotropy on travel times of the $P$ and $S$ waves and the phase velocities of Rayleigh waves, it is desirable to compare the relative perturbations. The travel times of both $P$ and $S$ waves are greater for the anisotropic model than for the isotropic. This increase is less than 3 per cent for $P$ waves and less than 4 per cent for $S$ waves. We should make it clear that the change is mainly due to the reduction of velocities in both the longitudinal and the transverse directions in the anisotropic zone. Since we are concerned with relative differences, this comparison is still valuable. The phase velocities of Rayleigh waves are affected more than the traveltime curves. The decrease of phase velocity in the anisotropic model is as high as 8 per cent in the range of periods where measurements were taken.

Phase velocities for the mantle Rayleigh and Love waves are now being measured with an accuracy of 0.5 per cent or better. Considering the relative effect of an anisotropic upper mantle on phase velocities, it should be possible to detect such a zone from dispersion of surface waves.

Acknowledgments. The theoretical work described in this paper was supported by grant AFAFOSR-25-63 of the Air Force Office of Scientific Research as part of the Advanced Research Projects Agency project Vela. The experimental portion was supported under grant DA-ARO(D)31-124-G216 of the Army Research Office (Durham).
We gratefully acknowledge the help of Carl Holmstrom in fabricating the models, and the help of Laszlo Lenches in preparing the manu* script. Shelton Alexander wrote the Fourier analy. sis program used in this study.

\section{REFERENCES}

Anderson, D. L., Elastic wave propagation in layered anisotropic media, J. Geophys. Res., 66, 2953-2963, 1961.

Ben-Menahem, A., and M. N. Toksöz, Source mechanism from spectra of long-period seismic surface-waves, I, The Mongolian earthquake of December 4, 1957, J. Geophys. Res., 67, 1943$1955,1962$.

Ewing, M., W. S. Jardetzky, and F. Press, Elastic Waves in Layered Media, McGraw-Hill Book Co., New York, 1957.

Gilbert, F., and S. L. Laster, Experimental in. vestigation of $P L$ modes in a single layer, $B$ ull Seismol. Soc. Am., 52, 59-66, 1962.

Harkrider, D. G., and D. L. Anderson, Computation of surface wave dispersion for multilayered anisotropic media, Bull. Seismol. Soc. Am., 52, 321-332, 1962.

Healy, J., and F. Press, Two-dimensional seismic models with continuously variable velocity depth and density functions, Geophysics, 25, 987-997, 1960.

Oliver, J. F., F. Press, and M. Ewing, Two-dimensional model seismology, Geophysics, 19, 202$219,1954$.

Phinney, R. A., Leaking modes in the crustal waveguide, I, The oceanic $P L$ wave, $J$. Geophys. Res., 66, 1445-1469, 1961.

(Manuscript received September 27, 1962; revised November 26, 1962.) 